\title{
Retained Foreign Body in Urinary Bladder Presenting as Acute Renal Failure
}

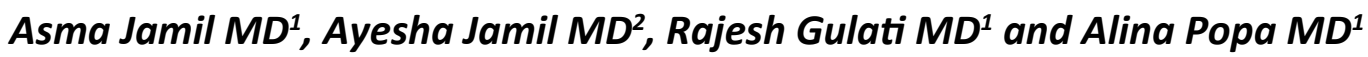 \\ ${ }^{1}$ Department of Internal Medicine, Riverside Community Hospital, California, USA \\ ${ }^{2}$ Department of Internal Medicine, St Mary Hospital, USA
}

*Corresponding author: Asma Jamil, MD, Department of Internal medicine, Riverside Community Hospital, 4445 Magnolia Ave, Riverside, California, 92501, USA, Tel: 951-235-9165

\section{Introduction}

Retained foreign bodies in urinary bladder can lead to stone formation. Urease splitting bacteria play a role in providing alkaline environment that lead to deposition of magnesium and calcium deposits. We described a severe presentation of a case which lead to permanent irreversible kidney injury ending up on permanent dialysis.

\section{Case Description}

A 57-year-old male with a past medical history of benign prostate hypertrophy (BPH) was admitted with altered mental status. Due to patient altered mental status history was obtained from family members. Patient used to live alone and was able to take care of his activities of daily living. He was found confused and covered in feces and urine by his family members. Last well known was 1 week ago. The patient was seen in hospital 5 years ago for urinary retention. A temporary Foley catheter was placed at that time and the patient was counseled to follow outpatient with the urology. However, patient never followed outpatient with urology. Physical exam revealed cut Foley at the external urethral meatus. Upon admission, lab findings were significant for severe uremia and acute renal failure with Bun of $192 \mathrm{mg} / \mathrm{dl}$, Creatinine $17.7 \mathrm{mg} / \mathrm{dl}$, and Potassium $7.3 \mathrm{mmol} / \mathrm{L}$. Other significantly abnormal labs included Hgb $5.7 \mathrm{~g} / \mathrm{dl}, \mathrm{Wbc} 17.2 \mathrm{k} / \mathrm{mm}^{3}$, Platelet $450 \mathrm{k} /$ $\mathrm{mm}^{3}$, Bicarb $9 \mathrm{mg} / \mathrm{dl}$. CT abdomen and pelvis without contrast showed $5.6 \times 6.0 \times 4.4 \mathrm{~cm}$ calcified stone with retained Foley in the urinary bladder (Figure 1). There was severe bilateral hydronephrosis and hydroureter.
Both kidneys demonstrated cortical thinning suggesting chronic renal disease. All these findings were suggestive of chronic urinary retention caused by BPH and by formation of stone around the retained foley. Urinalysis revealed cloudy urine, presence of leukocyte esterase, hematuria, pyuria, and bacteriuria. CT scan of the head without contrast did not reveal any acute intracranial abnormality.

The patient was admitted to ICU, intubated for airway protection and was started on broad-spectrum antibiotics e.g. cefepime. 3 units of blood were transfused. Emergent dialysis was done after temporary indwelling dialysis catheter placement, consecutively for 3 days. Blood cultures were sent which later grew Citrobacter koseri and Proteus mirabilis; both sensitive to cefepime and ceftriaxone.

He underwent open cystostomy with the removal of bladder stone and retained Foley enbloc with suprapubic catheter placement (Figure 2).

The patient had a retained Foley that later underwent calcification and a large bladder stone formation. He was able to urinate from half cut retained part of Foley which was placed 5 years ago. Once the Foley catheter lumen was occluded, he was unable to urinate and subsequently developed renal failure. During this hospitalization, telepsychology consult was placed regarding patient inability to make decision for himself and lack of insight regarding his disease. He was diagnose with cognitive impairment secondary to chronic kidney disease or from brain's microvascular disease. 


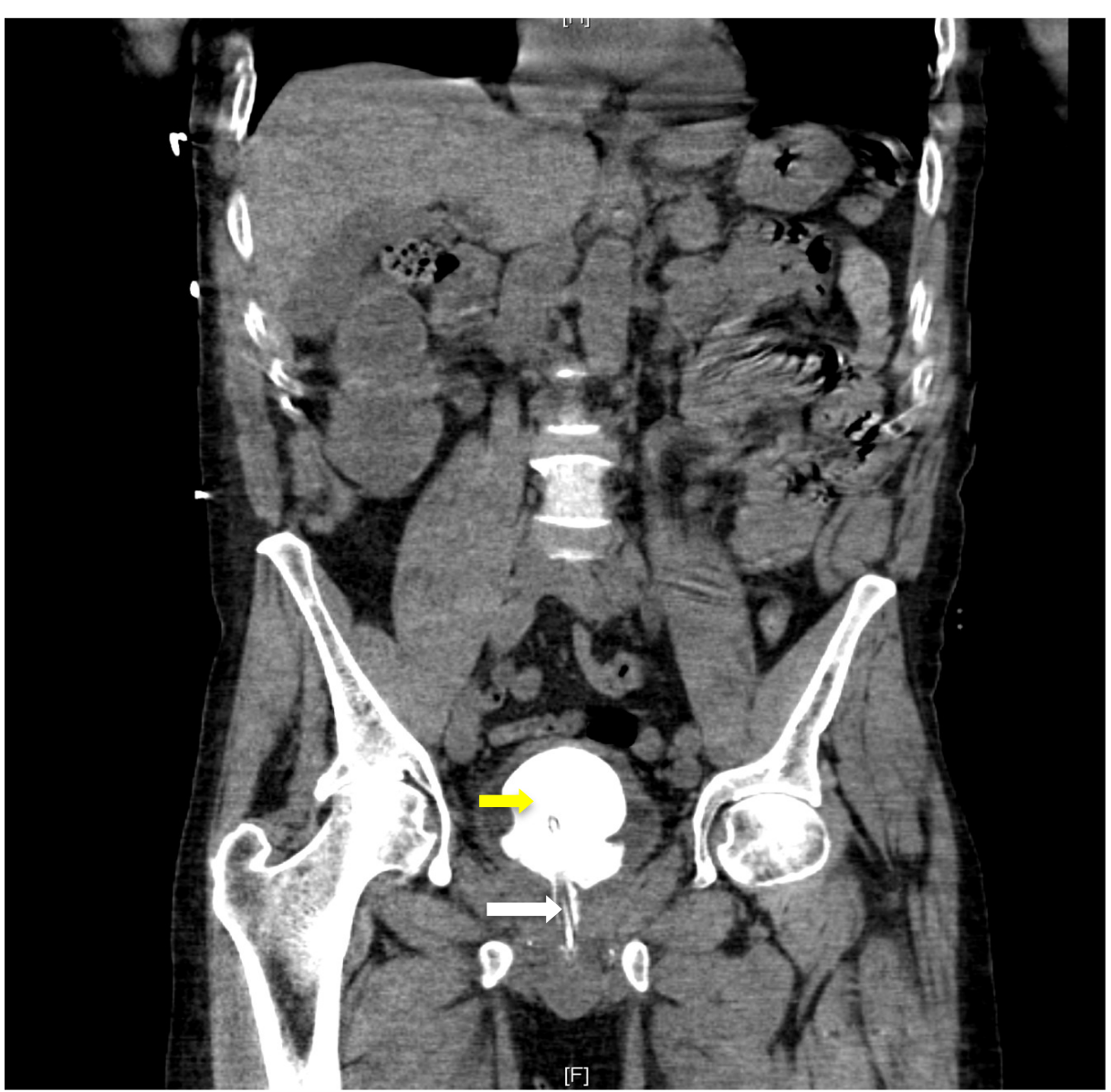

Figure 1: Yellow arrow represents bladder stone formed around the retained Foley. White arrow represents part of the retained Foley in the penile urethra.

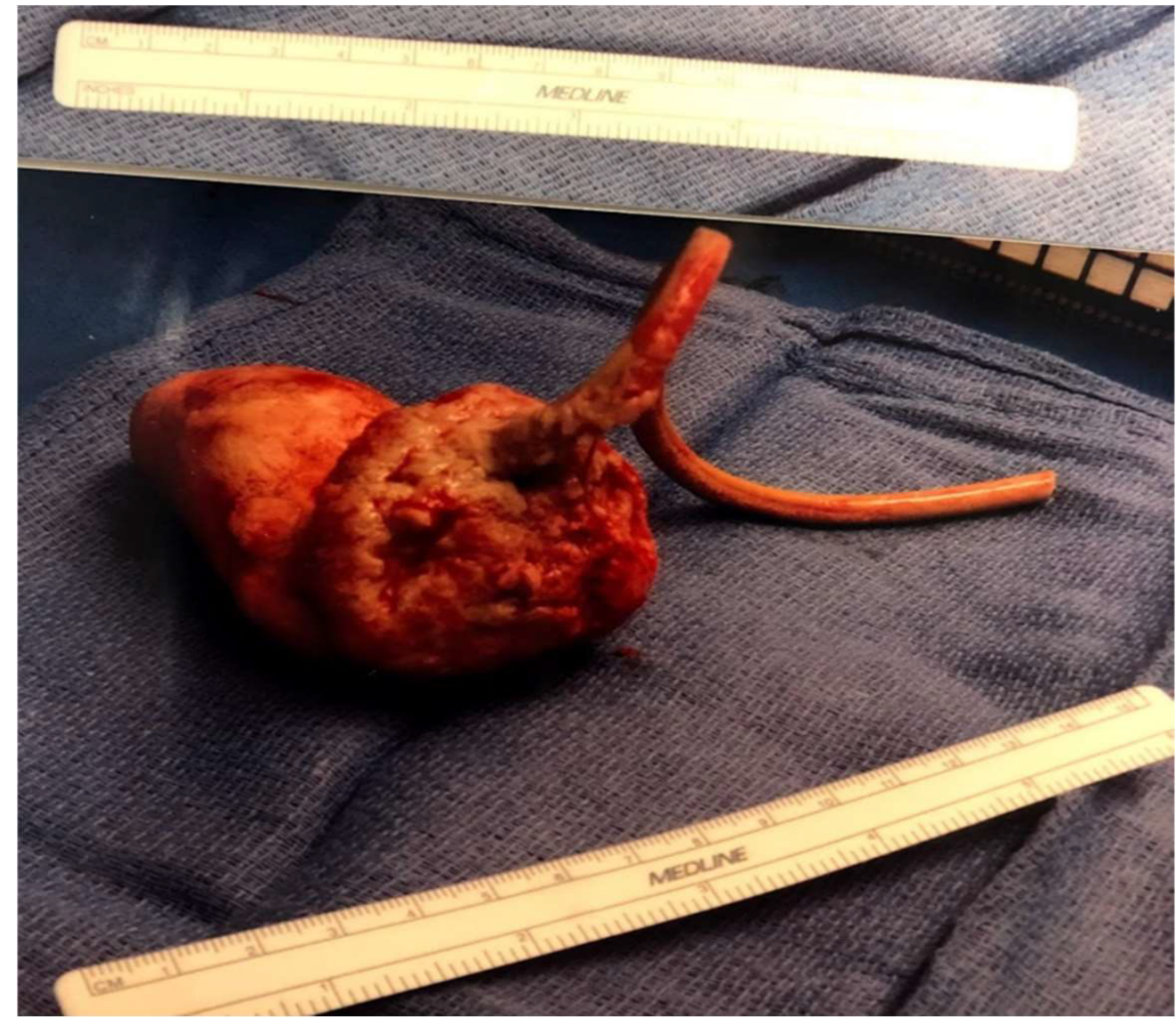

Figure 2: Large bladder stone with partially cut retained Foley. 
This patient developed serious post-obstructive renal failure and remained on dialysis even after emergent treatment. Suprapubic Foley was removed and he continued with Foley to drainage. AV fistula was constructed as a dialysis access route. The patient was later discharged to a rehabilitation center and dialysis chair was been arranged three days a week before discharge.

\section{Conclusion}

Retained foreign body in urinary bladder described in the literature includes Foleys catheter, burst pieces of Foley balloon, pencils, batteries, electric wires, hair clips, pieces of gauze, and pieces of gloves. The most common etiologies include psychosocial disorders, iatrogenic causes such as self-catheterization, and postop complications [1]. Encrustations around chronic indwelling Foley can lead to difficult removal and it can get stuck requiring surgical intervention. Retained Foley and its parts act as a nidus for stone formation. Urease forming bacteria e.g. Proteus form a biofilm around the Foley which leads to stone formation [2].

\section{Teaching Points}

1. Be mindful of Foley catheter complications and its implications.

2. History regarding urologic interventions in the past helps identify the cause of a patient's clinical presentation.

\section{Source of Funding}

None.

\section{Equal Author Contribution Statement}

All authors have equal contribution towards the preparation of this article.

\section{References}

1. Temiz MZ, Dincer M, Kandirali E (2015) Bladder stone secondary to retained foley catheter balloon in a woman. J Acad Res Med 5: 131-133.

2. Ho CC, Khandasamy $Y$, Singam P, Goh EH, Zainuddin ZM (2010) Encrusted and incarcerated urinary bladder catheter: What are the options? Libyan J Med 5. 